Università DI PISA

Dipartimento Di InFormaticA

TECHNICAL REPORT

\title{
Differentiated oligopolistic markets with concave cost functions via Ky Fan inequalities
}

Giancarlo Bigi Mauro Passacantando

12 January 2017

ADDRESS: Largo B.Pontecorvo, 3, 56127 Pisa, Italia.

TEL: +390502212700 FAX: +39050 2212726 



\title{
Differentiated oligopolistic markets with concave cost functions via Ky Fan inequalities
}

\author{
Giancarlo Bigi · Mauro Passacantando
}

\begin{abstract}
A Nash-Cournot model for oligopolistic markets with concave cost functions and a differentiated commodity is analysed. Equilibrium states are characterized through Ky Fan inequalities. Relying on the minimization of a suitable merit function, a general algorithmic scheme for solving them is provided. Two concrete algorithms are therefore designed that converge under suitable convexity and monotonicity assumptions. The results of some numerical tests on randomly generated markets are also reported.
\end{abstract}

Keywords Oligopoly - concave costs · Ky Fan inequalities - merit function · descent algorithm

\section{Introduction}

An oligopoly is a market structure with a small number of competing firms that produce the same kind of commodity. Since Cournot introduced and analysed the celebrated duopoly, models for oligopolies have been widely studied in economics (see, for instance, Bonanno (1990); Fudenberg and Tirole (1986); von Mouche and Quartieri (2016); Okuguchi and Szidarovszky (1990); Vives (1989)). The analysis of the competition typically exploits the tools of

G. Bigi

Dipartimento di Informatica, Università di Pisa

Largo B. Pontecorvo 3, 56127 Pisa, Italia

Tel.: +39-050-2213124

Fax: +39-050-2212726

E-mail: giancarlo.bigi@unipi.it

M. Passacantando

Dipartmento di Informatica, Università di Pisa

Largo B. Pontecorvo 3, 56127 Pisa, Italia

Tel.: +39-050-2212756

Fax: +39-050-2212726

E-mail: mauro.passacantando@unipi.it 
game theory to predict the behaviour of the firms (see, for instance, Friedman (1977)). In turn, if no cooperation between firms is allowed, game theoretic models can be formulated as variational problems (Harker, 1984; Murphy et al, 1982 ) and optimization techniques can be exploited to solve them (see, for instance, Flåm and Ben-Israel (1990); Harker (1984); Kolstad and Mathiesen (1991); Krawczyk and Uryasev (2000); Marcotte (1987); Nagurney (1988); Salant (1982)).

Generally, these game theoretic models shares two features that seem a bit restrictive. The costs increase at least linearly with the produced quantity, while usually the cost per unit does decrease as the production increases. As a consequence, costs should generally increase less than linearly. The commodity is supposed to be homogeneous, i.e., all the firms produce the identical product with even no slight difference, so that a unique unitary price is set for all the firms (by the market) through an inverse demand function. Commodities of different firms, though similar, have their own characteristics and are rarely identical. Moreover, firms generally try to differentiate their products from those of their competitors to improve their market share (see, for instance, Okuguchi and Szidarovszky (1990); Singh and Vives (1984)). As a consequence, the dynamics of the price should depend also on the considered firm. To the best of our knowledge, a first attempt to analyse product differentiation with concave costs can be found in Muu et al (2008) where piecewise linear cost functions have been considered modeling the market through mixed variational inequalities.

This paper provides another step in this direction. In Section 2 a NashCournot model for oligopolistic markets with concave quadratic cost functions and a differentiated commodity is introduced and formulated as Ky Fan inequalities. Section 3 describes a general algorithmic scheme for Ky Fan inequalities based on the minimization of a suitable merit function. Moreover, two concrete descent algorithms based on gap and D-gap functions are provided and their global convergence is deduced from the general scheme under suitable convexity and monotonicity assumptions. The uniqueness of the solution is also investigated. Finally, Section 4 provides numerical tests of the two algorithms for randomly generated markets. The sensitivity of the algorithms with respect to their parameters are reported and some market scenarios are analysed.

\section{Differentiated oligopoly models and Ky Fan inequalities}

Consider $n$ producers that supply the same kind of commodity to some market and compete over quantity in a noncooperative way. Each producer $i$ chooses a quantity $x_{i}$ to produce and supply at a $\operatorname{cost} c_{i}\left(x_{i}\right)$ for some given cost function $c_{i}: \mathbb{R}_{+} \rightarrow \mathbb{R}$. The largest unitary price at which the market requires a quantity $x_{i}$ of the commodity of producer $i$ depends also upon the quantities that the other producers supply. Since the commodities of the producers may differ in features and/or quality, then this price can be represented by an inverse 
demand function $p_{i}: \mathbb{R}_{+}^{n} \rightarrow \mathbb{R}$ that is peculiar of each producer. Therefore, the goal of producer $i$ is to maximize its profit

$$
f_{i}\left(x_{1}, \ldots, x_{n}\right)=x_{i} p_{i}\left(x_{1}, \ldots, x_{n}\right)-c_{i}\left(x_{i}\right)
$$

that is affected by the choices of the other producers. An equilibrium state of the market is reached when the levels of supply are such that no producer would increase its profit by changing its level while the other firms keep the same. Mathematically, $x^{*}=\left(x_{1}^{*}, \ldots, x_{n}^{*}\right) \in \mathbb{R}_{+}^{n}$ is an equilibrium state if and only if $x_{i}^{*} \in\left[0, T_{i}\right]$ and

$$
f_{i}\left(x^{*}\right) \geq f_{i}\left(y_{i}, x_{-i}^{*}\right)
$$

hold for all $y_{i} \in\left[0, T_{i}\right]$ and all $i=1, \ldots, n$, where $x_{-i}^{*}=\left(x_{j}^{*}\right)_{j \neq i}$ denotes the levels of supply of all the producers other than $i$ while $T_{i}>0$ denotes its maximum capacity of production. It is well-known that a feasible $x^{*}$ is an equilibrium state if and only if it solves the following Ky Fan inequalities

$$
f\left(x^{*}, y\right) \geq 0, \quad \forall y \in\left[0, T_{1}\right] \times \cdots \times\left[0, T_{n}\right],
$$

where $f: \mathbb{R}_{+}^{n} \times \mathbb{R}_{+}^{n} \rightarrow \mathbb{R}$ is the Nikaido-Isoda aggregate bifunction

$$
f(x, y)=\sum_{i=1}^{n}\left[f_{i}(x)-f_{i}\left(x_{-i}, y_{i}\right)\right],
$$

which measures the overall losses of the players when switching from level $x_{i}$ to $y_{i}$, one at a time while all the others stick to levels $x_{-i}$.

In classical models the cost functions $c_{i}$ are generally supposed to be affine (or even convex). As already remarked, often this assumption is not realistic and concave cost functions would sound more adequate. Therefore, in this paper the cost functions are supposed to be quadratic and concave, i.e.,

$$
c_{i}\left(x_{i}\right)=\ell_{i} x_{i}+q_{i} x_{i}^{2}
$$

for some $\ell_{i}>0$ and $q_{i}<0$ with $\ell_{i} \geq-2 T_{i} q_{i}$ so that $c_{i}$ is increasing on $\left[0, T_{i}\right]$.

The inverse demand functions are supposed to decrease linearly with the total production, i.e.,

$$
p_{i}\left(x_{1}, \ldots, x_{n}\right)=m_{i}-d_{i}\left(x_{1}+\cdots+x_{n}\right)
$$

for some $m_{i}>\ell_{i}$ and $d_{i}>-q_{i}$. Under these assumptions a unitary production guarantees a positive profit in a monopolistic setting, i.e., when $x_{-i}=0$. If all the rates $d_{i}$ are equal, the effect of the product differentiation does not meaningfully impact the dynamics of prices and moreover the equilibrium states coincide with the maxima of the sum of all the profit functions (see (Monderer and Shapley, 1996, Theorem 4.5)) just like in the case no differentiation occurs. Therefore, the rates $d_{i}$ are supposed to be not all equal.

Under all these assumptions the Nikaido-Isoda bifunction reads

$$
f(x, y)=\langle P x+Q y+r, y-x\rangle
$$


for $r=\left(\ell_{1}-m_{1}, \ldots, \ell_{n}-m_{n}\right) \in \mathbb{R}^{n}$ and the matrices

$$
P=\left[\begin{array}{cccc}
d_{1}+q_{1} & d_{1} & \cdots & d_{1} \\
d_{2} & \ddots & \ddots & \vdots \\
\vdots & \ddots & \ddots & d_{n-1} \\
d_{n} & \ldots & d_{n} & d_{n}+q_{n}
\end{array}\right], \quad Q=\left[\begin{array}{cccc}
d_{1}+q_{1} & 0 & \ldots & 0 \\
0 & \ddots & \ddots & \vdots \\
\vdots & \ddots & \ddots & 0 \\
0 & \ldots & 0 & d_{n}+q_{n}
\end{array}\right] .
$$

Notice that the nonzero entries of $Q$ are positive and hence it is a positive definite matrix. As a consequence, $f(x, \cdot)$ is a strongly convex function for any $x$. On the contrary, $f(\cdot, y)$ may not be concave as the symmetric part $\operatorname{sym}(P)=\left(P+P^{T}\right) / 2$ of $P$ is not necessarily positive semidefinite, but it is yet continuous. Furthermore the feasible region is a box, and hence convex and compact, so that the Ky Fan inequalities (1) admit at least one solution (see Bigi et al (2013)).

\section{A general descent algorithm for Ky Fan inequalities}

Consider the Ky Fan inequalities

$$
\text { find } x^{*} \in C \text { s.t. } f\left(x^{*}, y\right) \geq 0, \quad \forall y \in C \text {, }
$$

where $f: \mathbb{R}^{n} \times \mathbb{R}^{n} \rightarrow \mathbb{R}$ is any continuously differentiable bifunction satisfying $f(x, x)=0$ for all $x \in \mathbb{R}^{n}$, and $C \subseteq \mathbb{R}^{n}$ is a nonempty, closed and convex set. Ky Fan inequalities turned into optimization problems through suitable merit functions. Given a closed set $X \subseteq \mathbb{R}^{n}, \psi: \mathbb{R}^{n} \rightarrow \mathbb{R}$ is a merit function on $X$ for $(K F I)$ if it is nonnegative on $X$ and its zeros on $X$ coincide with the solutions of $(K F I)$, i.e, if it satisfies

$-\psi(x) \geq 0$ for any $x \in X$,

$-x^{*}$ solves (KFI) if and only if $x^{*} \in X$ and $\psi\left(x^{*}\right)=0$.

Therefore, (KFI) can be formulated as the global optimization problem

$$
\min \{\psi(x): x \in X\},
$$

where the set $X$ does not necessarily coincide with the feasible set $C$ of the Ky Fan inequalities. In order to solve it, $\psi$ is supposed to be continuously differentiable and to satisfy the following two properties:

(P1) $X$ is convex and the sublevel sets $\{x \in X: \psi(x) \leq c\}$ are bounded for any $c>0$.

(P2) There exist $\nu>0$ and continuous functions $d: \mathbb{R}^{n} \rightarrow \mathbb{R}^{n}$ and $\sigma: \mathbb{R}^{n} \rightarrow \mathbb{R}$ such that any $x \in X$ not solving (KFI) satisfies $x+d(x) \in X, \sigma(x)>0$ and

$$
\langle\nabla \psi(x), d(x)\rangle \leq-\nu \sigma(x) .
$$


Notice that (P2) implies that the global minima and the stationary points of $\psi$ over $X$ coincide even though $\psi$ is not necessarily convex. Therefore, descent techniques can be exploited to minimize it, or equivalently solve (KFI), just moving away form the current iterate $x^{k}$ along the direction $d\left(x^{k}\right)$ with a step provided by a standard linesearch rule.

\section{Descent algorithm}

Step 0. Choose $x^{0} \in X$, set $\eta \in(0, \nu), \delta \in(0,1), k=0$.

Step 1. If $\psi\left(x^{k}\right)=0$ then stop.

Step 2. Set $d^{k}=d\left(x^{k}\right), \sigma^{k}=\sigma\left(x^{k}\right)$ and compute the smallest non-negative integer $s$ such that

$$
\psi\left(x^{k}+\delta^{s} d^{k}\right) \leq \psi\left(x^{k}\right)-\eta \delta^{s} \sigma^{k}
$$

Step 3. Set $t_{k}=\delta^{s}, x^{k+1}=x^{k}+t_{k} d^{k}, k=k+1$ and go to Step 1 .

Notice that (P2) guarantees that the linesearch procedure at Step 2 is finite. Otherwise, the inequality

$$
\psi\left(x^{k}+\delta^{s} d^{k}\right)-\psi\left(x^{k}\right)>-\eta \delta^{s} \sigma^{k}
$$

would hold for all $s \in \mathbb{N}$. Taking the limit as $s \rightarrow+\infty$ would lead to

$$
\left\langle\nabla \psi\left(x^{k}\right), d^{k}\right\rangle \geq-\eta \sigma^{k}>-\nu \sigma^{k}
$$

in contradiction with (P2) since $x^{k}$ does not solve (KFI). The convexity of $X$ guarantees that the sequence $\left\{x^{k}\right\}$ is feasible and the theorem below shows its convergence properties.

Theorem 1 Suppose there exists a merit function $\psi$ on $X$ for (KFI) such that (P1) and (P2) hold. Then, either the descent algorithm stops at a solution to (KFI) after a finite number of iterations or it produces a bounded sequence $\left\{x^{k}\right\}$ such that any of its cluster points solves (KFI).

Proof If the algorithm stops at $x^{k}$ after a finite number of iterations at Step 2 , then $x^{k}$ solves (KFI) since $\psi$ is a merit function.

Now, suppose the algorithm generates an infinite sequence $\left\{x^{k}\right\}$. Such sequence is bounded since the sequence $\left\{\psi\left(x^{k}\right)\right\}$ is monotone decreasing and the sublevel sets of $\psi$ are bounded. Let $x^{*}$ be any cluster point of the sequence and let $\hat{x}^{\ell} \rightarrow x^{*}$ for some subsequence $\left\{\hat{x}^{\ell}\right\}$, i.e., $\hat{x}^{\ell}=x^{k_{\ell}}$ for some $k_{\ell} \uparrow+\infty$ as $\ell \uparrow+\infty$. The continuity of $d$ and $z$ guarantees $\hat{d}^{\ell}=d\left(\hat{x}^{\ell}\right) \rightarrow d\left(x^{*}\right)=d^{*}$ and $\hat{\sigma}^{\ell}=\sigma\left(\hat{x}^{\ell}\right) \rightarrow \sigma\left(x^{*}\right)=\sigma^{*}$.

By contradiction, suppose $x^{*}$ does not solve (KFI) and hence $\sigma^{*}>0$ by $(P 2)$. Since the sequence $\left\{\psi\left(x^{k}\right)\right\}$ is also bounded by below, it has a limit and thus

$$
\lim _{\ell \rightarrow \infty}\left[\psi\left(\hat{x}^{\ell}\right)-\psi\left(\hat{x}^{\ell+1}\right)\right]=0
$$


holds as well. Moreover, the stepsize rule guarantees

$$
\psi\left(\hat{x}^{\ell}\right)-\psi\left(\hat{x}^{\ell+1}\right) \geq \psi\left(x^{k_{\ell}}\right)-\psi\left(x^{k_{\ell}+1}\right) \geq \eta \hat{t}_{\ell} \hat{\sigma}^{\ell}>0
$$

for $\hat{t}_{\ell}=t_{k_{\ell}}$, Therefore, $\hat{t}_{\ell} \rightarrow 0$ as $\ell \rightarrow+\infty$ since $\sigma^{*}>0$. On the other side, the inequality

$$
\psi\left(\hat{x}^{\ell}+\hat{t}_{\ell} \delta^{-1} \hat{d}^{\ell}\right)-\psi\left(\hat{x}^{\ell}\right)>-\eta\left(\hat{t}_{\ell} \delta^{-1}\right) \hat{\sigma}^{\ell}
$$

holds for all $\ell \in \mathbb{N}$ by the linesearch procedure, while the mean value theorem guarantees the existence of some $\theta_{\ell} \in(0,1)$ such that

$$
\psi\left(\hat{x}^{\ell}+\hat{t}_{\ell} \delta^{-1} \hat{d}^{\ell}\right)-\psi\left(\hat{x}^{\ell}\right)=\left\langle\nabla \psi\left(\hat{x}^{\ell}+\theta_{\ell} \hat{t}_{\ell} \delta^{-1} \hat{d}^{\ell}\right), \hat{t}_{\ell} \delta^{-1} \hat{d}^{\ell}\right\rangle .
$$

Hence, the two inequalities together provide

$$
\left\langle\nabla \psi\left(\hat{x}^{\ell}+\theta_{\ell} \hat{t}_{\ell} \delta^{-1} \hat{d}^{\ell}\right), \hat{d}^{\ell}\right\rangle>-\eta \hat{\sigma}^{\ell} .
$$

Taking the limit, the inequalities

$$
\left\langle\nabla \psi\left(x^{*}\right), d^{*}\right\rangle \geq-\eta \sigma^{*}>-\nu \sigma^{*},
$$

contradict (P2). Therefore, $x^{*}$ solves (KFI).

Since the sequence $\left\{x^{k}\right\}$ is bounded, the above convergence theorem yields the existence of a solution of the Ky Fan inequalities as well.

Corollary 1 If there exists a merit function $\psi$ on $X$ for (KFI) such that properties (P1)-(P2) hold, then (KFI) admits at least one solution.

No assumptions other than smoothness are actually made on $f$ in the above results. Indeed, the tools for the convergence of the algorithm all lie in the properties $(P 1)$ and $(P 2)$. Concrete merit functions that satisfy them are given in the next subsections and suitable convexity and monotonicity assumptions on $f$ are required.

\subsection{Gap functions}

A possible measure for the quality of a candidate solution $x \in C$ of $(K F I)$ is the gap between zero and minimum value of $f(x, y)$ over all $y \in C$. To facilitate its computation, a regularization term is usually added leading to the value function

$$
\varphi_{\alpha}(x):=\sup \left\{-\left(f(x, y)+\alpha\|y-x\|^{2} / 2\right): y \in C\right\}
$$

for some $\alpha \in \mathbb{R}$ (see Bigi and Passacantando (2016)), also known as gap function. In order to guarantee that $\varphi_{\alpha}$ is a merit function, the parameter $\alpha$ must be related to the modulus of strong convexity of $f(x, \cdot)$. 
Definition 1 Given $\tau \in \mathbb{R}$, a function $g: \mathbb{R}^{n} \rightarrow \mathbb{R}$ is called $\tau$-convex on $C$ if any $u, v \in C$ and $t \in[0,1]$ satisfy

$$
g(t u+(1-t) v) \leq t g(u)+(1-t) g(v)-\frac{\tau}{2} t(1-t)\|u-v\|^{2} .
$$

If $\tau=0$, the above inequality provides the usual definition of convexity. If $\tau>0$, the inequality is strengthened and $g$ is called strongly convex. Indeed, $g$ is $\tau$-convex on $C$ if and only if $g(x)-\tau\|x\|^{2} / 2$ is convex on $C$ (see Vial (1983)). Similarly, $g$ is called $\tau$-concave on $C$ if $-g$ is $\tau$-convex on $C$.

If the functions $f(x, \cdot)$ are $\tau$-convex for any $x \in C$ and $\alpha+\tau>0$, then the objective function of the optimization problem in (3) is strongly convex and its minimum point is unique so that the map

$$
y_{\alpha}(x)=\arg \min \left\{-\left(f(x, y)+\alpha\|y-x\|^{2} / 2\right): y \in C\right\}
$$

is well defined. In order to guarantee good descent properties for $\varphi_{\alpha}$ also some monotonicity assumptions on $f$ are needed.

Definition $2 f$ is $\gamma$-c-monotone on $C$ if any $x, y \in C$ satisfy

$$
f(x, y)+\left\langle\nabla_{x} f(x, y), y-x\right\rangle \geq \gamma\|y-x\|^{2} .
$$

If $\gamma=0$, the above definition collapse to the concavity type condition introduced in Bigi et al (2009). Indeed, if the functions $f(\cdot, y)$ are $2 \gamma$-concave for any $y \in C$, then $f$ is $\gamma$-c-monotone on $C$. Indeed, the concavity of the function $g(x)=f(x, y)+\gamma\|x\|^{2}$ implies $g(y) \leq g(x)+\langle\nabla g(x), y-x\rangle$ that reads

$$
\gamma\|y\|^{2} \leq f(x, y)+\gamma\|x\|^{2}+\left\langle\nabla_{x} f(x, y)+2 \gamma x, y-x\right\rangle
$$

which is equivalent to the inequality in the above definition.

The main properties of $\varphi_{\alpha}$ are given below provided that the above convexity and monotonicity conditions hold for suitable moduli.

Theorem 2 Suppose that there exists $\tau \geq 0$ such that $f(x, \cdot)$ is $\tau$-convex for any $x \in C$. Then, the following hold for any $\alpha>-\tau$ :

a) $\varphi_{\alpha}$ is a merit function on $C$ for $(K F I)$;

b) $x^{*}$ solves $(K F I)$ if and only if $y_{\alpha}\left(x^{*}\right)=x^{*}$;

c) the map $y_{\alpha}$ is continuous on $\mathbb{R}^{n}$;

d) $\varphi_{\alpha}$ is continuously differentiable on $C$ and

$$
\nabla \varphi_{\alpha}(x)=-\nabla_{x} f\left(x, y_{\alpha}(x)\right)+\alpha\left(y_{\alpha}(x)-x\right) .
$$

Furthermore, suppose $f$ is $\gamma$-c-monotone for some $\gamma \in \mathbb{R}$. Then, the following hold whenever $\gamma+\tau / 2>0$ :

e) If $x \in C$ does not solve (KFI), then

$$
\left\langle\nabla \varphi_{\alpha}(x), y_{\alpha}(x)-x\right\rangle \leq-(\gamma+\tau / 2)\left\|y_{\alpha}(x)-x\right\|^{2}<0 .
$$


Proof See, for instance, Bigi and Passacantando (2016) for a) and d) and Konnov (2003) for $b$ ) and $c$ ).

e) If $x \in C$ does not solve (KFI), then the monotonicity and convexity assumptions of $f$ imply that the direction $y_{\alpha}(x)-x$ satisfies

$$
\begin{aligned}
\left\langle\nabla \varphi_{\alpha}(x), y_{\alpha}(x)-x\right\rangle= & -\left\langle\nabla_{x} f\left(x, y_{\alpha}(x)\right), y_{\alpha}(x)-x\right\rangle+\alpha\left\|y_{\alpha}(x)-x\right\|^{2} \\
\leq & f\left(x, y_{\alpha}(x)\right)-\gamma\left\|y_{\alpha}(x)-x\right\|^{2}+\alpha\left\|y_{\alpha}(x)-x\right\|^{2} \\
\leq & \left\langle\nabla_{y} f\left(x, y_{\alpha}(x)\right), y_{\alpha}(x)-x\right\rangle \\
& \quad-(\gamma+\tau / 2)\left\|y_{\alpha}(x)-x\right\|^{2}+\alpha\left\|y_{\alpha}(x)-x\right\|^{2} \\
\leq & -(\gamma+\tau / 2)\left\|y_{\alpha}(x)-x\right\|^{2} \\
< &
\end{aligned}
$$

where the third inequality follows from the first-order optimality condition for $y_{\alpha}(x)$.

Therefore, $\varphi_{\alpha}$ is a merit function on $C$ for (KFI) whenever $\alpha>-\tau$ is chosen and it satisfies (P1) provided that $C$ is bounded and (P2) for $\nu=\gamma+\tau / 2$, $d(x)=y_{\alpha}(x)-x$ and $\sigma(x)=\|d(x)\|^{2}$.

The Nikaido-Isoda aggregate bifunction (2) is $\tau$-convex for $\tau=2 \lambda_{\min }(Q)=$ $2 \min \left\{d_{i}+q_{i}: i=1, \ldots, n\right\}>0$, that is twice the minimum eigenvalue of $Q$, and it is also $\gamma$-c-monotone for $\gamma=\lambda_{\min }(\operatorname{sym}(P))$, that is the minimum eigenvalue of the symmetric part of $P$ (for which no simple formula is available).

\subsection{D-gap functions}

A class of merit functions on the whole space $\mathbb{R}^{n}$ is given by the difference of two gap functions, i.e., $\varphi_{\alpha \beta}(x):=\varphi_{\alpha}(x)-\varphi_{\beta}(x)$ with $\alpha<\beta$ (see Pappalardo et al (2016)). Good descent properties for $\varphi_{\alpha \beta}$ are guaranteed by another kind of monotonicity assumption.

Definition $3 f$ is $\mu-\nabla_{x y}$-monotone on $C$ if for any $x, y, z \in C$

$$
\left\langle\nabla_{x} f(x, y)-\nabla_{x} f(x, z), y-z\right\rangle \geq \mu\|y-z\|^{2} .
$$

If $\mu>0$, then $\nabla_{x y}$-monotonicity amounts to the strong monotonicity of $\nabla_{x} f(x, \cdot)$ and it has already been exploited in the framework of D-gap functions in Charitha (2013); Konnov and Pinyagina (2003).

If $f$ is $\mu-\nabla_{x y}$-monotone on $C$ and $f(x, \cdot)$ is convex, then $f$ is $\mu$-c-monotone on $C$. Indeed,

$$
\begin{aligned}
f(x, y)+\left\langle\nabla_{x} f(x, y), y-x\right\rangle & \geq f(x, y)+\left\langle\nabla_{x} f(x, x), y-x\right\rangle+\mu\|y-x\|^{2} \\
& =f(x, y)-\left\langle\nabla_{y} f(x, x), y-x\right\rangle+\mu\|y-x\|^{2} \\
& \geq \mu\|y-x\|^{2},
\end{aligned}
$$


where the last inequality is due to the convexity and the equality follows since $f(x, x)=0$ for all $x \in \mathbb{R}^{n}$ implies the equality of the two partial gradients above.

The joint exploitation of $\varphi_{\alpha}$ and $\varphi_{\beta}$ would suggest to rely on the direction

$$
r(x)=y_{\alpha}(x)-y_{\beta}(x) .
$$

However, $r(x)$ could be zero even when $x$ does not solve $(K F I)$. The gradient of $\varphi_{\alpha \beta}$ provides the additional term

$$
s(x)=\alpha\left(x-y_{\alpha}(x)\right)-\beta\left(x-y_{\beta}(x)\right)
$$

that is useful together with $r(x)$ to characterize Ky Fan inequalities.

The main properties of $\varphi_{\alpha \beta}$ are given below provided that the convexity and monotonicity conditions hold for suitable moduli.

Theorem 3 Suppose that there exists $\tau \geq 0$ such that $f(x, \cdot)$ is $\tau$-convex for any $x \in C$. Then, the following hold for any $\beta>\alpha>-\tau$ :

a) $\varphi_{\alpha \beta}$ is a merit function on $\mathbb{R}^{n}$ for $(K F I)$.

b) $x^{*}$ solves (KFI) if and only if $r\left(x^{*}\right)=s\left(x^{*}\right)=0$.

c) $\varphi_{\alpha \beta}$ is continuously differentiable on $\mathbb{R}^{n}$ and

$$
\nabla \varphi_{\alpha \beta}(x)=\nabla_{x} f\left(x, y_{\beta}(x)\right)-\nabla_{x} f\left(x, y_{\alpha}(x)\right)-s(x) .
$$

d) If $C$ is bounded, then the sublevel sets $\left\{x \in \mathbb{R}^{n}: \varphi_{\alpha \beta}(x) \leq c\right\}$ are bounded for all $c>0$.

Moreover, suppose $f$ is $\mu-\nabla_{x y}$-monotone on $\mathbb{R}^{n}$ for some $\mu \in \mathbb{R}$ and $\nabla_{x} f(x, \cdot)$ is Lipschitz on $\mathbb{R}^{n}$ with modulus $L>0$ for any $x \in \mathbb{R}^{n}$. Then, the following hold whenever $\mu+\tau>0$ and $0<\rho \leq \min \left\{1 /(\mu+\tau),(\mu+\tau) /(\mu+\tau+L)^{2}\right\}$ :

e) If $x \in \mathbb{R}^{n}$ does not solve (KFI), then

$$
\left\langle\nabla \varphi_{\alpha \beta}(x), r(x)+\rho s(x)\right\rangle \leq-2^{-1}(\mu+\tau)(\|r(x)\|+\rho\|s(x)\|)^{2}<0 .
$$

Proof

a) By definition $\varphi_{\alpha}(x) \geq \varphi_{\beta}(x)$ for any $x \in \mathbb{R}^{n}$, so that $\varphi_{\alpha \beta}$ is non-negative everywhere. If $x^{*}$ solves (KFI), then $\varphi_{\alpha}\left(x^{*}\right)=\varphi_{\beta}\left(x^{*}\right)=0$ by Theorem $2 a$ ). Viceversa, if $\varphi_{\alpha \beta}\left(x^{*}\right)=0$, then

$$
\begin{aligned}
0=\varphi_{\alpha \beta}\left(x^{*}\right)=\varphi_{\alpha}\left(x^{*}\right)-\varphi_{\beta}\left(x^{*}\right) \geq & -f\left(x^{*}, y_{\beta}\left(x^{*}\right)\right)-\alpha\left\|y_{\beta}\left(x^{*}\right)-x\right\|^{2} / 2 \\
& +f\left(x^{*}, y_{\beta}\left(x^{*}\right)\right)+\beta\left\|y_{\beta}\left(x^{*}\right)-x^{*}\right\|^{2} / 2 \\
= & (\beta-\alpha)\left\|y_{\beta}\left(x^{*}\right)-x^{*}\right\|^{2} / 2 .
\end{aligned}
$$

implies that $x^{*}=y_{\beta}\left(x^{*}\right)$, hence $x^{*}$ solves (KFI) by Theorem $2 b$ ).

b) If $x^{*}$ solves (KFI), then $x^{*}=y_{\alpha}\left(x^{*}\right)=y_{\beta}\left(x^{*}\right)$ by Theorem $\left.2 b\right)$ and hence $r\left(x^{*}\right)=s\left(x^{*}\right)=0$. Viceversa, if $r\left(x^{*}\right)=0$, then $y_{\alpha}\left(x^{*}\right)=y_{\beta}\left(x^{*}\right)$ so that $0=s\left(x^{*}\right)=(\alpha-\beta)\left(x^{*}-y_{\alpha}\left(x^{*}\right)\right)$ implies $x^{*}=y_{\alpha}\left(x^{*}\right)$, i.e., $x^{*}$ solves $(K F I)$.

c) It is a straightforward consequence of Theorem $2 c$ ). 
d) See Zhang and Han (2009).

e) The first-order optimality conditions guarantee that $y_{\alpha}(x)$ and $y_{\beta}(x)$ satisfy

$$
\begin{aligned}
& \left\langle\nabla_{y} f\left(x, y_{\alpha}(x)\right)+\alpha\left(y_{\alpha}(x)-x\right), y_{\beta}(x)-y_{\alpha}(x)\right\rangle \geq 0, \\
& \left\langle\nabla_{y} f\left(x, y_{\beta}(x)\right)+\beta\left(y_{\beta}(x)-x\right), y_{\alpha}(x)-y_{\beta}(x)\right\rangle \geq 0,
\end{aligned}
$$

so that summing up the two inequalities provides

$$
\left\langle\nabla_{y} f\left(x, y_{\beta}(x)\right)-\nabla_{y} f\left(x, y_{\alpha}(x)\right)+s(x), r(x)\right\rangle \geq 0 .
$$

The convexity of $f(x, \cdot)$ implies that $\nabla_{y} f(x, \cdot)$ is strongly monotone (see (Hiriart-Urruty and Lemarchal, 1993, Theorem 6.1.2)), and therefore the above inequality guarantees also

$$
\langle s(x), r(x)\rangle \geq\left\langle\nabla_{y} f\left(x, y_{\alpha}(x)\right)-\nabla_{y} f\left(x, y_{\beta}(x)\right), r(x)\right\rangle \geq \tau\|r(x)\|^{2} .
$$

Let $d=r(x)+\rho s(x)$. Therefore, the following chain of equalities and inequalities holds

$$
\begin{aligned}
\left\langle\nabla \varphi_{\alpha \beta}(x), d\right\rangle= & \left\langle\nabla_{x} f\left(x, y_{\beta}(x)\right)-\nabla_{x} f\left(x, y_{\alpha}(x)\right), r(x)\right\rangle \\
& +\rho\left\langle\nabla_{x} f\left(x, y_{\beta}(x)\right)-\nabla_{x} f\left(x, y_{\alpha}(x)\right), s(x)\right\rangle \\
& -\langle s(x), r(x)\rangle-\rho\|s(x)\|^{2} \\
\leq & -\mu\|r(x)\|^{2}+\rho\left\langle\nabla_{x} f\left(x, y_{\beta}(x)\right)-\nabla_{x} f\left(x, y_{\alpha}(x)\right), s(x)\right\rangle \\
& -\langle s(x), r(x)\rangle-\rho\|s(x)\|^{2} \\
\leq & -(\mu+\tau)\|r(x)\|^{2} \\
& +\rho\left\langle\nabla_{x} f\left(x, y_{\beta}(x)\right)-\nabla_{x} f\left(x, y_{\alpha}(x)\right), s(x)\right\rangle-\rho\|s(x)\|^{2} \\
\leq & -(\mu+\tau)\|r(x)\|^{2}+\rho L\|r(x)\|\|s(x)\|-\rho\|s(x)\|^{2} \\
= & (\mu+\tau)(\|r(x)\|+\rho\|s(x)\|)^{2} / 2 \\
& -(\sqrt{\mu+\tau}\|r(x)\|-\sqrt{\rho}\|s(x)\|)^{2} / 2+[(\gamma+\tau) \rho-1] / 2 \\
& +\sqrt{\rho}[\sqrt{\rho}(\mu+\tau+L)-\sqrt{\mu+\tau}]\|r(x)\|\|s(x)\| \\
\leq & -(\|r(x)\|+\rho\|s(x)\|)^{2} / 2 \\
< & 0
\end{aligned}
$$

where the first inequality is due to the $\nabla_{x y}$-monotonicity of $f$, the second to (4), the third to the Lipschitz continuity of $\nabla_{x} f(x, \cdot)$, the fourth to the assumption on $\rho$ and the last to $b$ ).

Therefore, $\varphi_{\alpha \beta}$ is a merit function on $\mathbb{R}^{n}$ for (KFI) whenever $\beta>\alpha>-\tau$ are chosen and it satisfies (P1) provided that $C$ is bounded and (P2) for $\nu=(\mu+\tau) / 2, d(x)=r(x)+\rho s(x)$ and $\sigma(x)=(\|r(x)\|+\rho\|s(x)\|)^{2}$.

The resulting descent algorithm had already been proposed in Charitha (2013); Konnov and Pinyagina (2003) just for $\mu>0$ and $\tau=0$, while it is enough to require $\mu+\tau>0$ as shown above. 
The Nikaido-Isoda aggregate bifunction (2) is $\mu$ - $\nabla_{x y}$-monotone for $\mu=$ $\lambda_{\min }(\operatorname{sym}(P-Q))$, that is the minimum eigenvalue of the symmetric part of $P-Q$, while the partial gradient map $\nabla_{x} f(x, \cdot)$ is Lipschitz with $L=\|P-Q\|$.

\subsection{Uniqueness of the solution}

The convexity and monotonicity conditions of the previous subsections yield also the uniqueness of the solution of the Ky Fan inequalities.

Theorem 4 Suppose there exists $\tau \geq 0$ such that $f(x, \cdot)$ is $\tau$-convex for any $x \in C$. Then, there exists a unique solution of (KFI) provided that any of the following statements holds:

a) $f$ is twice continuously differentiable and $\gamma$-c-monotone on $C$ for some $\gamma \in \mathbb{R}$ satisfying $\gamma+\tau / 2>0$

b) $f$ is $\mu-\nabla_{x y}$-monotone on $\mathbb{R}^{n}$ for some $\mu \in \mathbb{R}$ with $\mu+\tau>0$.

Proof

a) Let $\theta=\gamma+\tau / 2$ and consider $g(x, y)=f(x, y)+\theta\left(\|x\|^{2}-\|y\|^{2}\right) / 2$. The following chain of equalities and inequalities

$$
\begin{aligned}
& \left\langle\nabla_{x} g(x, y)+\nabla_{y} g(x, y), y-x\right\rangle= \\
& \left\langle\nabla_{x} f(x, y)+\nabla_{y} f(x, y), y-x\right\rangle-\theta\|y-x\|^{2}= \\
& f(x, y)+\left\langle\nabla_{x} f(x, y), y-x\right\rangle-\left[f(x, y)+\left\langle\nabla_{y} f(x, y), x-y\right\rangle\right]-\theta\|y-x\|^{2} \geq \\
& \gamma\|y-x\|^{2}-f(x, x)+\tau\|y-x\|^{2} / 2-\theta\|y-x\|^{2}=0
\end{aligned}
$$

follows from the $\gamma$-c-monotonicity of $f$ and the $\tau$-convexity of $f(x, \cdot)$. As a consequence, setting $y(t)=(1-t) x+t y$ for any given pair $x, y \in C$, the function $\omega(t)=\left\langle\nabla_{x} g(x, y(t))+\nabla_{y} g(x, y(t)), y(t)-x\right\rangle$ is non-negative on $[0,1]$ and $\omega(0)=0$ so that

$$
\begin{aligned}
0 & \leq \omega^{\prime}(0)=\left\langle\nabla_{x y} f(x, y)+\nabla_{y y} f(x, y), y-x\right\rangle-\theta\|y-x\|^{2} \\
& =\langle y-x, J F(x)(y-x)\rangle-\theta\|y-x\|^{2}
\end{aligned}
$$

holds for any $x, Y \in C$, where $J F(x)$ denotes the Jacobian of the map $F(z)=\nabla_{y} f(z, z)$ at $x$. Therefore, $F$ is strongly monotone on $C$ and the variational inequality

$$
\text { find } x^{*} \in C \text { s.t. }\left\langle F\left(x^{*}\right), y-x^{*}\right\rangle \geq 0, \quad \forall y \in C
$$

has a unique solution (see Facchinei and Pang (2003)). Since the above variational inequality is equivalent to (KFI) (see Castellani and Giuli (2010); Konnov (2003)), the thesis follows. 
b) The auxiliary problem principle (see (Bigi and Passacantando, 2016, Corollary 3.3)) guarantees that (KFI) is equivalent to the Ky Fan inequalities with $f_{-\tau}$. Furthermore, $f_{-\tau}$ is $(\mu+\tau)-\nabla_{x y}$-monotone as

$$
\begin{aligned}
\left\langle\nabla_{x}\right. & \left.f_{-\tau}(x, y)-\nabla_{x} f_{-\tau}(x, z), y-z\right\rangle \\
& =\left\langle\nabla_{x} f(x, y)-\nabla_{x} f(x, z), y-z\right\rangle+\langle(-\tau)(x-y)+\tau(x-z), y-z\rangle \\
& =\left\langle\nabla_{x} f(x, y)-\nabla_{x} f(x, z), y-z\right\rangle+\tau\|y-z\|^{2} \\
& \geq(\mu+\tau)\|y-z\|^{2} .
\end{aligned}
$$

Since $\mu+\tau>0, f_{-\tau}$ is strongly $\nabla_{x y}$-monotone on $C$. As strong $\nabla_{x y^{-}}$ monotonicity implies strong monotonicity (Bigi and Passacantando, 2015b, Theorem 3.1), the existence of a unique solution follows (see, for instance, (Bigi and Passacantando, 2016, Corollary 3.4)).

Twice differentiability of $f$ can be dropped from point $a$ ) if $\gamma$-c-monotonicity is replaced by the $2 \gamma$-concavity of the functions $f(\cdot, y)$ (Bigi and Passacantando, 2016, Corollary 3.7).

\section{Numerical tests}

The two algorithms described in the previous section have been implemented in MATLAB R2015b to compute the equilibria of oligopolistic markets through the resolution of the Ky Fan inequalities (1) where $f$ is the Nikaido-Isoda aggregate bifunction (2).

First, some preliminary tests have been run to analyse the impact of the parameters on the efficiency of the two algorithms. Afterwards, a few market scenarios with different kinds of producers and different levels of product differentiation are analysed in details.

Random instances have been produced using MATLAB generators in such a way that all the coefficients and bounds are pseudorandom values drawn from the uniform distribution over given intervals.

The efficiency of the algorithms has been evaluated relying just on the number of iterations. In fact, the computation of the values of the gap ad Dgap functions $\varphi_{\alpha}$ and $\varphi_{\alpha \beta}$, which is generally the heaviest task as it aumounts to solving optimization problems, can be done by closed-form formulas. Indeed, the optimization problem in (3) can be decomposed into $n$ one-dimensional quadratic optimization problems over intervals. In details, the decomposition

$$
\begin{aligned}
\varphi_{\alpha}(x)= & \sum_{i=1}^{n} \max _{y_{i} \in\left[0, T_{i}\right]}\left[f_{i}\left(y_{i}, x_{-i}\right)-\alpha\left(y_{i}-x_{i}\right)^{2} / 2\right]-\sum_{i=1}^{n} f_{i}(x) \\
= & \sum_{i=1}^{n} \max _{y_{i} \in\left[0, T_{i}\right]}\left\{y_{i}\left[m_{i}-d_{i}\left(y_{i}+\sum_{j \neq i} x_{j}\right)\right]-\ell_{i} y_{i}-q_{i} y_{i}^{2}-\alpha\left(y_{i}-x_{i}\right)^{2} / 2\right\} \\
& -\sum_{i=1}^{n}\left\{x_{i}\left[m_{i}-d_{i} \sum_{i=1}^{n} x_{i}\right]-\ell_{i} x_{i}-q_{i} x_{i}^{2}\right\}
\end{aligned}
$$


entails

$$
\left[y_{\alpha}(x)\right]_{i}=\min \left\{T_{i}, \max \left\{0, \frac{m_{i}-\ell_{i}+\alpha x_{i}-d_{i} \sum_{j \neq i} x_{j}}{2\left(d_{i}+q_{i}\right)+\alpha}\right\}\right\}, \quad i=1, \ldots, n
$$

\subsection{Preliminary tests}

The two concrete descent algorithms with the choices given in Section 3.1 and 3.2 will be briefly referred to as the gap and D-gap algorithm. Instances have been produced for $n=5$ taking the coefficients $m_{i}, \ell_{i}$ and the bounds $T_{i}$ in the ranges $[150,250],[30,50]$ and $[3,7]$, respectively. The coefficients $d_{i}$ have been drawn from $[5,20]$ and sorted in increasing order, while the coefficients $q_{i}$ have been obtained by dividing each $d_{i}$ by a pseudorandom number in $[-10,-2.5]$ and then sorting the outcome in decreasing order. In this way a smaller decrease rate for prices matches a smaller discount from a linear growth of costs. Each instance has been validated only if $\ell_{i}+2 q_{i} T_{i} \geq 0$, so that cost functions $c_{i}$ are increasing on $\left[0, T_{i}\right]$, and $\mu+\tau>5$, since the D-gap algorithm is unlikely to perform well when $\mu+\tau$ is close to zero (see Bigi and Passacantando (2015a)).

Pseudorandom starting points have been taken and the stopping criterion $\left\|x^{k}-y_{\alpha}\left(x^{k}\right)\right\|<10^{-3}$ has been used relying on Theorem $2 b$ ), since the values of gap functions are not scale free. A first set of tests suggested to set $\alpha=1$, $\delta=0.5$ and $\eta=0.8 \nu$ for the gap algorithm while $\alpha=1, \beta=100, \delta=0.5$ and $\eta=0.2 \nu$ for the D-gap algorithm.

Intensive computational tests have been carried out to analyse the sensitivity of the algorithms with respect to different values of the parameters $\delta, \eta$, $\alpha$ and $\rho$ by running the algorithms for 1000 random instances for each choice. Table 1 reports the sensitivity with respect to $\delta$ and $\eta$ : each row gives the average number of iterations that have been performed. The results suggest that the choice of these parameters has a weak impact on the performance of the algorithms.

Table 2 reports the performance of the gap algorithm when different values of $\alpha$ are chosen. The results show that a good performance is obtained when $\alpha$ is neither too close to the minimum value $-\tau$ nor too large.

Finally, the sensitivity of the D-gap algorithm with respect to $\rho$ has been tested. Table 3 suggests that the best performance is obtained when it is equal to the maximum value $\rho_{\max }=\min \left\{1 /(\mu+\tau),(\mu+\tau) /(\mu+\tau+L)^{2}\right\}$ that can be set (see Theorem 3).

As the gap algorithm generally perfoms better than the D-gap algorithm, the test for the market scenarios have been carried out just exploiting the former. 
Table 1 Sensitivity of the algorithms with respect to $\delta$ and $\eta$.

\begin{tabular}{cccc}
\hline$\delta$ & $\eta$ & $\begin{array}{c}\text { gap } \\
\text { (\# iter.) }\end{array}$ & $\begin{array}{c}\text { D-gap } \\
\text { (\# iter.) }\end{array}$ \\
\hline 0.3 & $0.2 \nu$ & 18.77 & 39.27 \\
0.3 & $0.4 \nu$ & 18.62 & 39.27 \\
0.3 & $0.6 \nu$ & 18.53 & 39.27 \\
0.3 & $0.8 \nu$ & 18.47 & 39.35 \\
0.5 & $0.2 \nu$ & 17.54 & 37.97 \\
0.5 & $0.4 \nu$ & 17.41 & 38.00 \\
0.5 & $0.6 \nu$ & 17.33 & 38.07 \\
0.5 & $0.8 \nu$ & 17.34 & 38.12 \\
0.7 & $0.2 \nu$ & 25.60 & 40.28 \\
0.7 & $0.4 \nu$ & 24.35 & 40.21 \\
0.7 & $0.6 \nu$ & 20.50 & 39.93 \\
0.7 & $0.8 \nu$ & 19.30 & 39.86 \\
\hline
\end{tabular}

Table 2 Sensitivity of the gap algorithm with respect to $\alpha$.

\begin{tabular}{cc}
\hline$\alpha$ & $\begin{array}{c}\text { gap } \\
\text { (\# iter.) }\end{array}$ \\
\hline$-0.5 \tau$ & 23.93 \\
0 & 17.99 \\
1 & 17.62 \\
5 & 22.61 \\
10 & 22.49 \\
\hline
\end{tabular}

Table 3 Sensitivity of the D-gap algorithm with respect to $\rho$.

\begin{tabular}{cr}
\hline$\rho$ & $\begin{array}{r}\text { D-gap } \\
\text { (\# iter.) }\end{array}$ \\
\hline $0.2 \rho_{\max }$ & 177.24 \\
$0.4 \rho_{\max }$ & 89.96 \\
$0.6 \rho_{\max }$ & 61.14 \\
$0.8 \rho_{\max }$ & 46.80 \\
$\rho_{\max }$ & 38.37 \\
\hline
\end{tabular}

\subsection{Market scenarios}

A few scenarios have been generated to try and analyse the effect of product differentiation on oligopolistic markets. The decrease rates $d_{i}$ of prices have been considered as a measure for the differentiation from the average commodity: higher differentiation calls for smaller rates as the price of a commodity with an high level of differentiation is likely to be less affected by the total production available on the market.

Scenarios with 5 producers have been considered, supposing that 2 produce a commodity of high quality while the other 3 produce a commodity of lower 
quality. Notice that here quality is meant merely with respect to the technical manufacture of the commodity and not to those features that may differentiate it. Quality has been addressed through higher costs and maximum prices $m_{i}$. Higher costs have been given through both larger coefficients $l_{i}$ and also smaller maximum percentage discounts $\left|q_{i}\right| T_{i} / l_{i}$ from a linear growth. In particular, the coefficients $l_{i}$ and $m_{i}$ have been chosen in the ranges $[30,50]$ and $[250,350]$ with a maximim percentage discount between $5 \%$ and $10 \%$ for the "high quality" producers $h 1$ and $h 2$, while in the ranges $[15,25]$ and $[150,250]$ with a maximim percentage discount between $10 \%$ and $30 \%$ for the "lower quality" producers $l 1, l 2$ and $l 3$.

Scenarios in which both "high" and "lower quality" producers do and don't differentiate their commodity much (coefficients in the ranges $[1,4]$ and $[5,10]$ ) have been considered with capacity bounds in the ranges $[3,7]$ for the former and $[5,11]$ for the latter. Table 4 reports the average results of 1000 random instances for each pair of choices, where "work load" denotes the percentage of the maximum capacity $T_{i}$ that is actually produced. The table suggests that the most beneficial situation for the overall market is when all the producers highly differentiate their commodity, while the behaviour of one type of producers does not affect much the profit of the other.

Table $4 T_{h 1}, T_{h 2} \in[3,7], T_{l 1}, T_{l 2}, T_{l 3} \in[5,11]$

\begin{tabular}{|c|c|c|c|c|c|c|c|}
\hline & $d_{h}$ & $d_{l}$ & $h 1$ & $h 2$ & $l 1$ & $l 2$ & $l 3$ \\
\hline & {$[5,10]$} & {$[1,4]$} & 6.21 & 7.68 & 2.70 & 3.21 & 3.62 \\
\hline \multirow[t]{4}{*}{ differentiation } & {$[1,4]$} & {$[1,4]$} & 2.33 & 3.15 & 2.01 & 2.69 & 3.33 \\
\hline & {$[5,10]$} & {$[5,10]$} & 6.66 & 8.30 & 6.23 & 7.49 & 8.73 \\
\hline & {$[1,4]$} & {$[5,10]$} & 2.95 & 3.54 & 6.18 & 7.31 & 8.54 \\
\hline & {$[5,10]$} & {$[1,4]$} & 91.60 & 53.77 & 99.93 & 99.48 & 97.40 \\
\hline \multirow[t]{4}{*}{ work load } & {$[1,4]$} & {$[1,4]$} & 100.00 & 100.00 & 99.97 & 99.17 & 96.48 \\
\hline & {$[5,10]$} & {$[5,10]$} & 99.11 & 92.36 & 80.99 & 46.54 & 20.47 \\
\hline & {$[1,4]$} & {$[5,10]$} & 100.00 & 100.00 & 77.56 & 48.13 & 19.36 \\
\hline & {$[5,10]$} & {$[1,4]$} & 14.62 & 8.47 & 26.57 & 25.41 & 24.94 \\
\hline \multirow[t]{4}{*}{ market share } & {$[1,4]$} & {$[1,4]$} & 15.03 & 14.84 & 24.02 & 23.22 & 22.88 \\
\hline & {$[5,10]$} & {$[5,10]$} & 23.68 & 22.23 & 30.27 & 16.35 & 7.47 \\
\hline & {$[1,4]$} & {$[5,10]$} & 24.50 & 23.78 & 27.98 & 16.89 & 6.86 \\
\hline & {$[5,10]$} & {$[1,4]$} & 332.90 & 133.00 & 834.97 & 668.86 & 549.23 \\
\hline \multirow[t]{3}{*}{ profit } & {$[1,4]$} & {$[1,4]$} & 946.24 & 787.85 & 946.84 & 730.77 & 569.26 \\
\hline & {$[5,10]$} & {$[5,10]$} & 611.70 & 417.86 & 398.89 & 156.39 & 52.77 \\
\hline & {$[1,4]$} & {$[5,10]$} & 1052.76 & 959.41 & 362.31 & 167.54 & 48.79 \\
\hline
\end{tabular}

A scenario in which "high quality" producers differentiate their commodity meaningfully and the other don't but prefer to increase their production capacities $T_{i}$ has been considered as well. Table 5 reports the average results of 1000 random instances for capacities $T_{i}$ in the ranges $[3,7][5,11]$ and $[9,21]$, suggesting that the effect of their increase is small and not necessarily positive, in any case definitely not valuable as product differentation. 
Table $5 T_{h 1}, T_{h 2} \in[3,7], d_{h 1}, d_{h 2} \in[1,4], d_{l 1}, d_{l 2}, d_{l 3}, \in[5,10]$.

\begin{tabular}{lcrrrrr}
\hline & $T_{l}$ & $h 1$ & $h 2$ & $l 1$ & $l 2$ & $l 3$ \\
\hline differentiation & & 2.90 & 3.50 & 6.09 & 7.19 & 8.46 \\
\hline \multirow{3}{*}{ work load } & {$[3,7]$} & 100.00 & 100.00 & 96.19 & 85.06 & 60.32 \\
& {$[5,11]$} & 100.00 & 100.00 & 84.92 & 62.03 & 30.94 \\
& {$[9,21]$} & 100.00 & 100.00 & 49.15 & 23.17 & 8.11 \\
\hline \multirow{3}{*}{ market share } & {$[3,7]$} & 13.08 & 12.87 & 30.04 & 25.93 & 18.08 \\
& {$[5,11]$} & 15.69 & 15.29 & 33.75 & 23.53 & 11.74 \\
& {$[9,21]$} & 24.99 & 24.32 & 31.04 & 14.38 & 5.27 \\
\hline \multirow{3}{*}{ profit } & {$[3,7]$} & 484.40 & 451.87 & 399.07 & 278.21 & 148.21 \\
& {$[5,11]$} & 637.29 & 581.04 & 446.33 & 245.28 & 93.45 \\
& {$[9,21]$} & 1126.73 & 1012.60 & 401.71 & 144.74 & 39.92 \\
\hline
\end{tabular}

Acknowledgements We would like to thank Marco Castellani and Massimiliano Giuli for providing background material for the proof of Theorem $4 \mathrm{a}$ ).

The authors are members of the Gruppo Nazionale per l'Analisi Matematica, la Probabilità e le loro Applicazioni (GNAMPA - National Group for Mathematical Analysis, Probability and their Applications) of the Istituto Nazionale di Alta Matematica (INdAM National Institute of Higher Mathematics).

\section{References}

Bigi G, Passacantando M (2015a) D-gap functions and descent techniques for solving equilibrium problems. Journal of Global Optimization 62(1):183-203

Bigi G, Passacantando M (2015b) Twelve monotonicity conditions arising from algorithms for equilibrium problems. Optimization Methods and Software 30(2):323-337

Bigi G, Passacantando M (2016) Auxiliary problem principles for equilibria. Optimization DOI 10.1080/02331934.2016.1227808

Bigi G, Castellani M, Pappalardo M (2009) A new solution method for equilibrium problems. Optim Methods Softw 24(6):895-911

Bigi G, Castellani M, Pappalardo M, Passacantando M (2013) Existence and solution methods for equilibria. European Journal of Operational Research 227(1):1-11

Bonanno G (1990) General equilibrium theory with imperfect competition. Journal of Economic Surveys 4(4):297-328

Castellani M, Giuli M (2010) On equivalent equilibrium problems. Journal of Optimization Theory and Applications 147(1):157-168

Charitha C (2013) A note on D-gap functions for equilibrium problems. Optimization 62(2):211-226

Facchinei F, Pang JS (2003) Finite-dimensional variational inequalities and complementarity problems : vol. 1. Springer series in operations research, Springer, New York

Flåm SD, Ben-Israel A (1990) A continuous approach to oligopolistic market equilibrium. Operations Research 38(6):1045-1051 
Friedman J (1977) Oligopoly and the theory of games. Advanced textbooks in economics, North-Holland Pub. Co.

Fudenberg D, Tirole J (1986) Dynamic Models of Oligopoly. Harwood Academic Publishers

Harker PT (1984) A variational inequality approach for the determination of oligopolistic market equilibrium. Mathematical Programming 30(1):105-111

Hiriart-Urruty JB, Lemarchal C (1993) Convex analysis and minimization algorithms. I. , Fundamentals. Grundlehren der mathematischen Wissenschaften, Springer, Berlin, New York

Kolstad CD, Mathiesen L (1991) Computing Cournot-Nash equilibria. Operations Research 39(5):739-748

Konnov I (2003) Application of the proximal point method to nonmonotone equilibrium problems. Journal of Optimization Theory and Applications 119(2):317-333

Konnov IV, Pinyagina OV (2003) D-gap functions for a class of equilibrium problems in Banach spaces. Comput Methods Appl Math 3(2):274-286

Krawczyk JB, Uryasev S (2000) Relaxation algorithms to find nash equilibria with economic applications. Environmental Modeling \& Assessment 5(1):6373

Marcotte P (1987) Algorithms for the network oligopoly problem. Journal of the Operational Research Society 38(11):1051-1065

Monderer D, Shapley LS (1996) Potential games. Games and Economic Behavior 14(1):124 - 143

von Mouche P, Quartieri F (eds) (2016) Equilibrium theory for Cournot oligopolies and related games. Springer Series in Game Theory, Springer, [Cham], essays in honour of Koji Okuguchi

Murphy FH, Sherali HD, Soyster AL (1982) A mathematical programming approach for determining oligopolistic market equilibrium. Mathematical Programming 24(1):92-106

Muu LD, Nguyen VH, Quy NV (2008) On nash-cournot oligopolistic market equilibrium models with concave cost functions. Journal of Global Optimization 41(3):351-364

Nagurney A (1988) Algorithms for oligopolistic market equilibrium problems. Regional Science and Urban Economics 18(3):425 - 445

Okuguchi K, Szidarovszky F (1990) The theory of oligopoly with multi-product firms, Lecture Notes in Economics and Mathematical Systems, vol 342. Springer-Verlag, Berlin

Pappalardo M, Mastroeni G, Passacantando M (2016) Merit functions: a bridge between optimization and equilibria. Annals of Operations Research 240(1):271-299

Salant SW (1982) Imperfect competition in the international energy market: A computerized nash-cournot model. Operations Research 30(2):252-280

Singh N, Vives X (1984) Price and quantity competition in a differentiated duopoly. The RAND Journal of Economics 15(4):546-554

Vial JP (1983) Strong and weak convexity of sets and functions. Mathematics of Operations Research 8(2):231-259 
Vives X (1989) Cournot and the oligopoly problem. European Economic Review 33(2-3):503-514

Zhang LP, Han JY (2009) Unconstrained optimization reformulations of equilibrium problems. Acta Math Sin (Engl Ser) 25(3):343-354 